\title{
Aneurysm of the Internal Mammary Artery with Cystic Medial Degeneration
}

\author{
Yuji Okura ${ }^{1}$, Takashi Kawasaki ${ }^{2}$, Tohru Hiura ${ }^{3}$, Hiroshi Seki ${ }^{4}$ and Hirofumi Saito ${ }^{5}$
}

\begin{abstract}
We herein report the histopathology of a rare case of an idiopathic internal mammary artery aneurysm in a 61-year-old asymptomatic woman. Chest radiography during an annual medical check-up incidentally revealed the aneurysm, which was initially mistaken for a mediastinal tumor. Given that a rupture of the aneurysm could have been life-threatening, it was removed surgically, and found to possess a paper-thin arterial wall with cystic medial degeneration.
\end{abstract}

Key words: aneurysm, internal mammary artery, cystic medial degeneration

(Intern Med 51: 2355-2359, 2012)

(DOI: 10.2169/internalmedicine.51.8139)

\section{Introduction}

Approximately 6.7 million Japanese people undergo chest radiography annually as part of routine medical checkups (1). Cardiovascular disease is sometimes detected during screens for lung cancer using chest radiography, but an aneurysm of the internal mammary artery (IMA) is seldom reported. A survey of the literature published in English indicated that 40 cases of IMA aneurysm have been reported since 1973, two-thirds of which were iatrogenic pseudoaneurysms following sternotomy or the placement of central venous catheters or pacemaker leads (2-6). Aneurysms of the IMA caused by non-iatrogenic etiologies such as vasculitis (e.g., Kawasaki disease (7), polyarteritis nodosa, and systemic lupus erythematosus), connective tissue diseases (e.g., Marfan syndrome (8) and Ehlers-Danlos syndrome (9)), type 1 neurofibrinomatosis (10), fibromuscular dysplasia (11), atherosclerosis, and idiopathic causes are very rare. Given that IMA aneurysms often rupture and cause hemothorax, their existence can be life-threatening (8-10). We herein report a case of idiopathic IMA aneurysm in which detection of an abnormal shadow following chest radiography enabled diagnosis and preventive surgery before a life-threatening event.

\section{Case Report}

A 61-year-old asymptomatic woman underwent chest radiography as part of an annual medical check-up at a clinic. The film showed a mass in the right upper anterior mediastinum (Fig. 1). Serial chest radiography in the same patient during the previous two years had failed to identify any tumors. She was referred to our hospital for further examination.

The patient had no history of trauma, catheter insertion, or mediastinal irradiation. She was $163 \mathrm{~cm}$ in height and 55 $\mathrm{kg}$ in weight and had none of the phenotypes associated with Marfan syndrome. Her blood pressure was normal, and there was no evidence of either a heart murmur or any other abnormal heart sounds. Blood tests revealed no signs of dyslipidemia, diabetes, or inflammation. She had chronic kidney disease with renal cysts, and her estimated glomerular filtration rate was $27 \mathrm{~mL} / \mathrm{min} / 1.73 \mathrm{~m}^{2}$. She had no vascular bruit in her abdomen. Her electrocardiogram and echocardiogram were both normal.

Ultrasonography revealed a throbbing aneurysm in the right subclavicular region, and computer tomography confirmed the presence of an aneurysm of the right IMA (Fig. 2). Digital subtraction angiography indicated the pres-

\footnotetext{
${ }^{1}$ Department of Cardiology, Niigata Cancer Center Hospital, Japan, ${ }^{2}$ Department of Pathology, Niigata Cancer Center Hospital, Japan, ${ }^{3}$ Department of Respiratory Medicine, Niigata Cancer Center Hospital, Japan, ${ }^{4}$ Department of Radiology, Niigata Cancer Center Hospital, Japan and ${ }^{5}$ Department of Cardiovascular Surgery, Niigata Medical Center Hospital, Japan Received for publication May 9, 2012; Accepted for publication June 11, 2012 Correspondence to Dr. Yuji Okura, okuray@niigata-cc.jp
} 
ence of a globular aneurysm with a tortuous pattern of arteries flowing into and out of the IMA (Fig. 3). The diameter of the origin of the right IMA was larger than that of the left IMA. The patient's coronary arteriography was normal. Systemic examinations with multi-slice computer tomography and positron emission tomography showed neither insidious cardiovascular abnormalities nor inflammation.

The aneurysm was removed after double ligation of the distal and proximal parts of the mammary artery (Fig. 4).

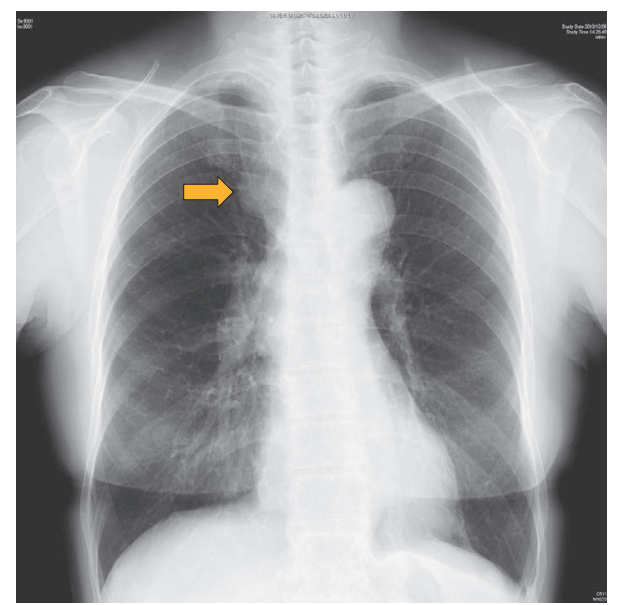

Figure 1. A chest X-ray taken at the time of hospital admission. The film showed a mass in the right upper anterior mediastinum (arrow).
The collapsed aneurysm measured $20 \mathrm{~mm}$ in diameter. A histopathological analysis of the aneurysm and the proximal inflow artery revealed that the resected aneurysm had an extremely thin wall with hyalinized fibrosis that was lacking normal arterial components (Fig. 5). Neither inflammatory infiltrates nor lipid components were observed. The inflow artery comprised two regions, one in which normal components of the artery were fairly well preserved (almost normal) and the other (aneurysmal region) in which the normal components were lost (Fig. 6). Degeneration and fragmentation of the elastic layers and loss of smooth muscle cells were evident in the media of the aneurysmal border (Fig. 6A-C). These areas accumulated acid mucopolysaccharide, which showed a blue color following colloidal iron staining (Fig. 6D). These histopathological findings were consistent with cystic medial degeneration (CMD) rather than atherosclerosis. The patient was discharged 10 days after the operation and has been in good health for over one year since the surgery.

\section{Discussion}

Non-iatrogenic IMA aneurysms are so rare that there is limited information about their management and prognosis. In this case, we anticipated the rapid growth of the aneurysm because chest radiographs from previous years showed no signs of an aneurysm. The paper-thin wall of the resected aneurysm suggested that its rupture was likely to have been
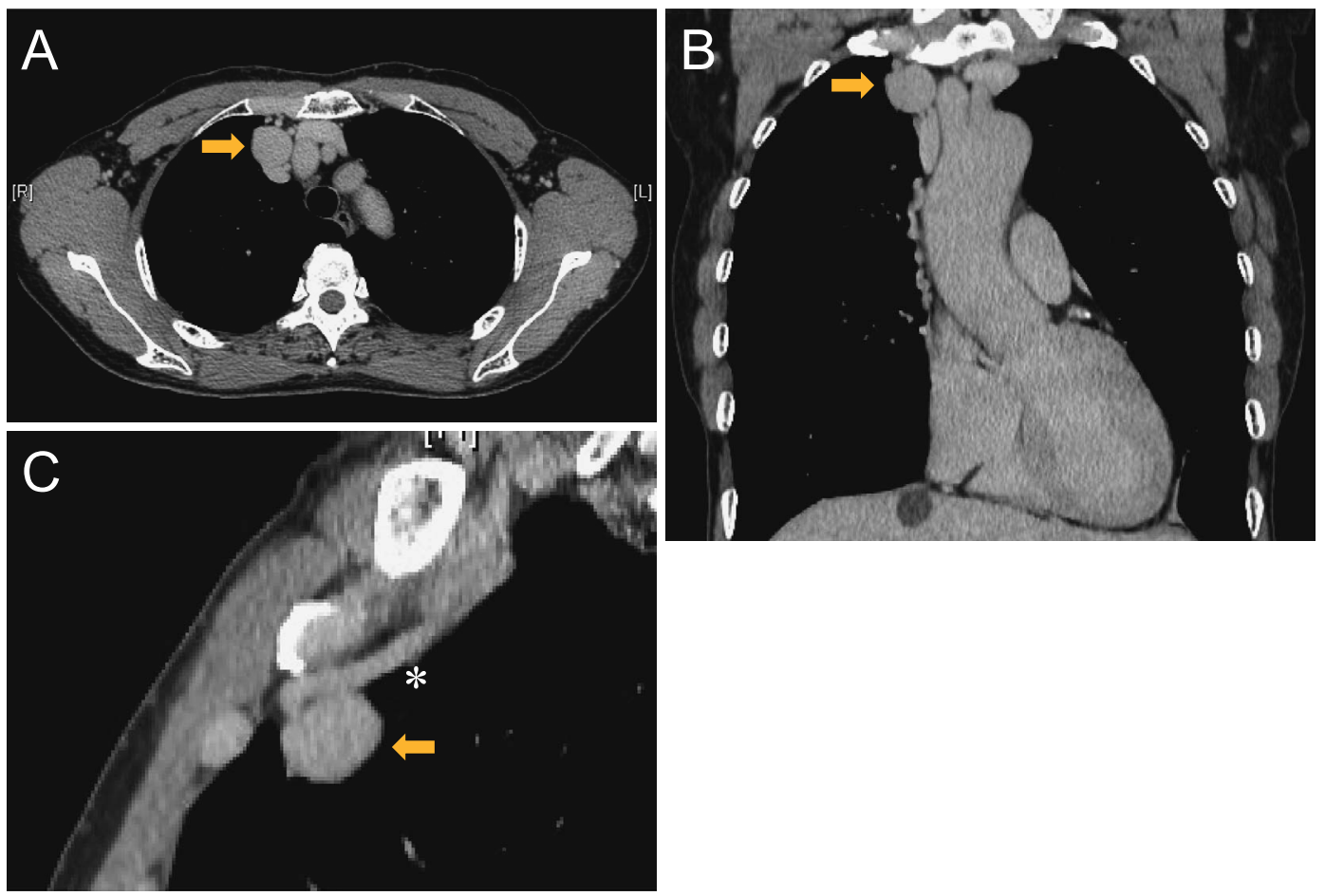

Figure 2. Computer tomography images in the (A) transverse plane, (B) coronal plane, and (C) sagittal plane. An aneurysm (arrow) in the IMA was observed near the brachiocephalic artery. The internal mammary artery entering the aneurysm was dilated (asterisk). 


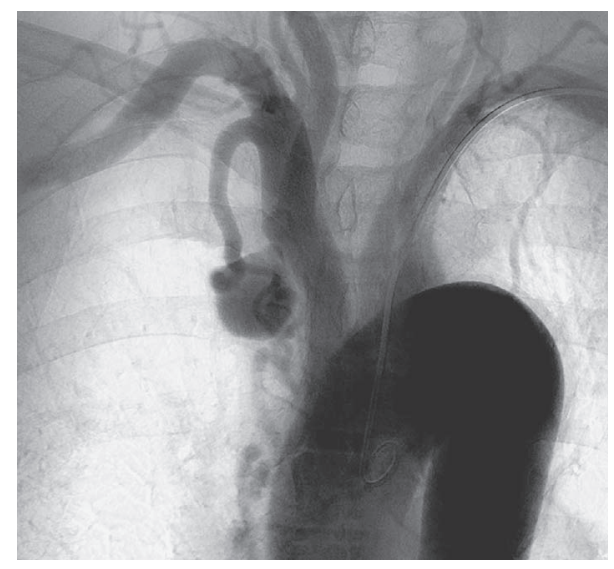

Figure 3. Intra-aortic digital subtraction angiography revealed a globular aneurysm with tortuous arteries entering and leaving the IMA. The diameter of the origin of the right IMA was larger than that of the left IMA.
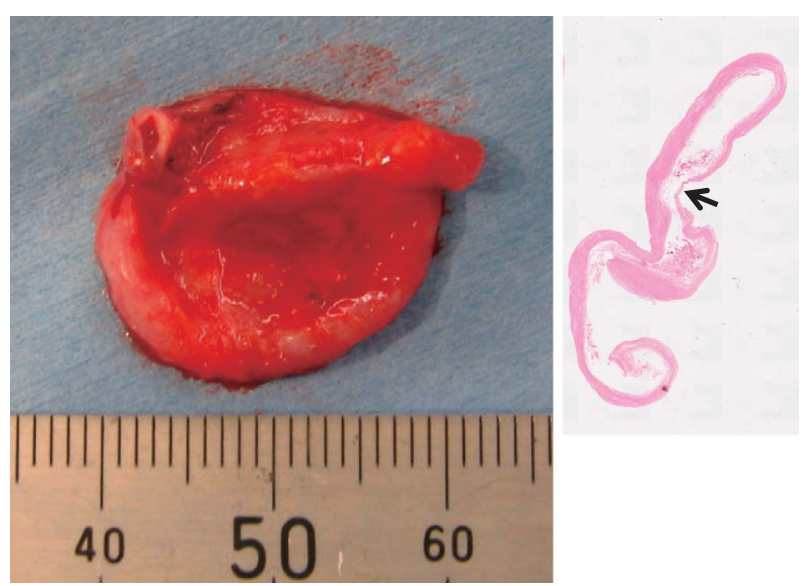

Figure 5. The resected IMA aneurysm. The collapsed aneurysm (left) had a diameter of $20 \mathrm{~mm}$. A whole mount of the aneurysm (right) stained with Hematoxylin and Eosin staining revealed that it had a very thin wall (cross-section).

imminent had it not been surgically removed. The rupture of IMA aneurysms may be fatal, although patients with ruptured IMA aneurysms occasionally arrive at the hospital alive. Hemothorax with shock is the most common initial manifestation of an IMA aneurysm (8-10).

Prior to surgery for complete ligation, we considered coil embolization as an alternative management option. Coil embolization is rapidly becoming the treatment of choice for arteriovenous fistulas and small aneurysms, although only six manuscripts have reported the short-term outcome after treatment of IMA aneurysms $(3-6,8,10)$. Four of these six studies assessed the recovery from iatrogenic pseudoaneurysms, which are slightly different from our case. Although coil embolization is less invasive, we were not confident that it would completely ablate the aneurysm and ensure longterm survival of the patient. Moreover, the procedure does not provide any histological information. We thus chose complete ligation for surgical removal of the aneurysm in

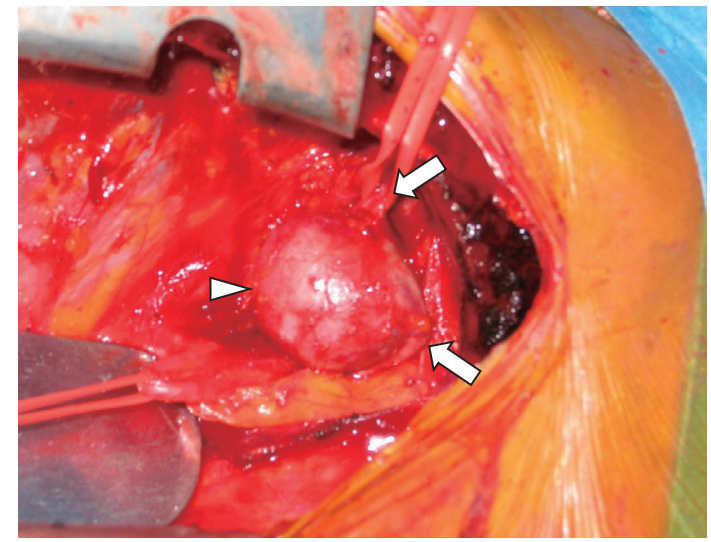

Figure 4. Intraoperative observations. The arrowhead indicates the aneurysm, and the two arrows indicate the arteries feeding into and out of the IMA.

the present case.

Case reports of non-iatrogenic IMA aneurysms are very rare. Those we could identify were associated with vasculitis (7), hereditary connective tissue diseases $(8,9)$, type 1 neurofibrinomatosis (10), fibromuscular dysplasia (11), or atherosclerosis. Clinically, our case seems to be idiopathic because the patient had neither an inflammatory nor connective tissue disease. The aorta of the patient appeared normal following angiography (Fig. 3) and enhanced computer tomography. Positron emission tomography detected neither inflammation nor atherosclerosis in the arterial walls. A histological analysis indicated similarities to the symptoms of CMD, but not atherosclerosis. Classically, a histopathology related to CMD is associated with Marfan syndrome, although CMD is now also often observed following aortic dissection or resectioning of aneurysms, regardless of the presence of more specific phenotypic characteristics of Marfan syndrome $(12,13)$. With changes in life expectancy over recent years, CMD is increasingly prevalent in healthy elderly people $(12,13)$. However, CMD is more common in the aorta than in the IMA.

We did not classify the primary etiology of this case as involving either aging or atherosclerosis. First, our patient was only 61 years old, and we failed to identify any clinical signs of atherosclerosis, such as hypertension, left ventricular hypertrophy, or carotid plaques. Moreover, she had very few risk factors for atherosclerosis. Second, diagnostic imaging demonstrated that, with the exception of her right IMA, her systemic arteries were free from substantial atherosclerosis, inflammation, and other anomalies. Third, although her right IMA was abnormal, the left IMA was intact. The laterality of the IMA suggested that the patient had no systemic conditions, including those related to aging and atherosclerosis, but that the IMA was a focal abnormality. Fourth, IMAs are not prone to atherosclerosis.

We herein reported a very rare case of idiopathic IMA aneurysm with CMD. Serial chest radiographs suggested rapid growth of the aneurysm because it was not detected in prior radiographs. Given that the paper-thin wall of an aneu- 


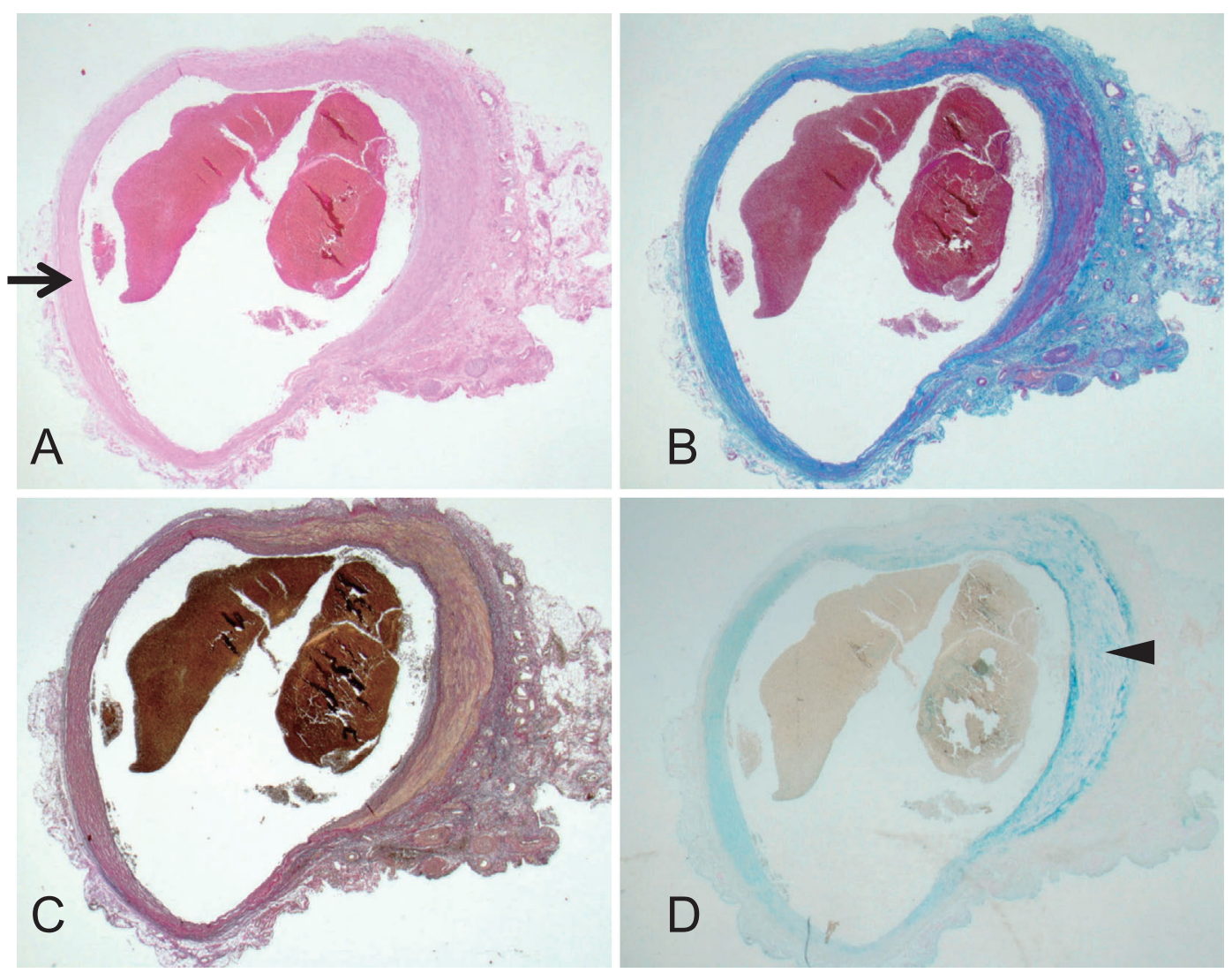

Figure 6. The results of the histopathological and immunohistochemical analysis of the inflow artery. Sections were stained using (A) Hematoxylin and Eosin staining, (B) Azan-Mallory, (C) Elastic van Gieson, and (D) colloidal iron staining. The arrow in (A) indicates the aneurysmal region that had lost components found in a normal elastic artery. The concentric laminae of elastic tissue normally sandwiched between successive layers of smooth muscle cells have been replaced by hyalinized fibrous tissue. As shown by the arrowhead in (D), fibrous tissue and acidic mucopolysaccharides replaced many of the normal components even in the apparently intact region. The examination of the border of the IMA revealed that less smooth muscle was found closer to the aneurysm than in other parts of the IMA (Original magnification, $\times 12.5$ ).

rysm could have caused it to rupture, it needed to be removed cautiously. There is a possibility that this was a case of a sporadic class of aneurysm potentially resulting from mutations that affect connective tissue, thereby weakening the structural integrity of the arterial wall. It seems that careful follow-up is needed to detect a possible aneurysm in other arteries, and the possibility of an increased risk of arterial disease in this patient's offspring cannot be excluded.

The authors state that they have no Conflict of Interest (COI).

\section{References}

1. Summary of cancer screening in 2006. Home page of Japan Cancer Society. http://www.jcancer.jp/about_cancer/kenshin/index.html (Accessed May 1, 2012).

2. Martin A, Ross BA, Braimbridge MV. Peristernal wiring in closure of median sternotomy. False aneurysm of the internal mammary artery. J Thorac Cardiovasc Surg 66: 145-146, 1973.

3. Chemelli AP, Chemelli-Steingruber IE, Bonaros N, et al. Coil embolization of internal mammary artery injured during central vein catheter and cardiac pacemaker lead insertion. Eur J Radiol 71:
269-274, 2009.

4. Namai A, Sakurai M, Akiyama M. Poststernotomy pseudoaneurysm of the internal mammary artery. Gen Thorac Cardiovasc Surg 56: 344-346, 2008.

5. Jenkins NP, Gray H. Pseudoaneurysm of the right internal thoracic artery. N Engl J Med 358: 1603, 2008.

6. Williams RJ, Ablett M. Embolisation of a false aneurysm of the right internal mammary artery complicating routine sternotomy. Eur J Cardiothorac Surg 19: 85-86, 2001.

7. Ishiwata S, Nishiyama S, Nakanishi S, et al. Coronary artery disease and internal mammary artery aneurysms in a young woman: possible sequelae of Kawasaki disease. Am Heart J 120: 213-217, 1990.

8. Rose JF, Lucas LC, Bui TD, Mills JL Sr. Endovascular treatment of ruptured axillary and large internal mammary artery aneurysms in a patient with Marfan syndrome. J Vasc Surg 53: 478-482, 2011.

9. Phan TG, Sakulsaengprapha A, Wilson M, Wing R. Ruptured internal mammary artery aneurysm presenting as massive spontaneous haemothorax in a patient with Ehlers-Danlos syndrome. Aust N Z J Med 28: 210-211, 1998.

10. Kim SJ, Kim CW, Kim S, et al. Endovascular treatment of a ruptured internal thoracic artery pseudoaneurysm presenting as a massive hemothorax in a patient with type I neurofibromatosis. Car- 
Intern Med 51: 2355-2359, 2012 DOI: 10.2169/internalmedicine.51.8139

diovasc Intervent Radiol 28: 818-821, 2005.

11. Connery CP, Cramer SF, Cheeran D. Multiple aneurysms of the internal thoracic artery. Ann Thorac Surg 59: 1561-1563, 1995.

12. Guo D, Hasham S, Kuang SQ, et al. Familial thoracic aortic aneu- rysms and dissections: genetic heterogeneity with a major locus mapping to 5q13-14. Circulation 103: 2461-2468, 2001.

13. Isselbacher EM. Thoracic and abdominal aortic aneurysms. Circulation 111: 816-828, 2005.

(C) 2012 The Japanese Society of Internal Medicine http://www.naika.or.jp/imonline/index.html 